Trauma Berufskrankh 2015 • [Suppl 2]:

17:247-248

DOI 10.1007/s10039-015-0029-1

Online publiziert: 19. Mai 2015

๑) Springer-Verlag Berlin Heidelberg 2015

\title{
P.A. Grützner
}

Klinik für Unfallchirurgie und Orthopädie, BG-Unfallklinik Ludwigshafen, Ludwigshafen, Deutschland

\section{Begrüßungsansprache}

strukturen darauf achten, dass hier alle Interessen adressiert sind. Daher wollen wir diesen Behandlungsauftrag der gesetzlichen Unfallversicherung auch im Programm widerspiegeln. Ich hoffe, dass uns das gelingt, und wir bitten auch in diesem Jahr wieder um Ihre Rückmeldung. Wir hoffen, dass sich die UMed in diesem Sinne immer weiterentwickelt. Als ich letzte Woche die Anmeldezahlen erfahren habe, war ich begeistert und froh, dass wir mit diesem Programm eine so große Resonanz gefunden haben.

Die Überwindung der Sektorengrenzen steht nun auch auf der Agenda der gesetzlichen Krankenversicherung. Ist das das Vorbild der DGUV, dem sich die Politik nun angenommen hat? Beispielsweise steht ein sog. Innovationsfond zur Förderung innovativer sektorenübergreifender Versorgungsformen im Raum. Dabei sollen auch Mittel für die Versorgungsforschung zur Verfügung gestellt werden. Das klingt sehr gut und bei dem Begriff „Fond“ hat man auch gleich den Eindruck, dass dieser mehr Geld ins System spült. Ob das so ist, sei dahingestellt.

Allerdings werden gleich auch berechtigte Bedenken geäußert. Niedergelassene Ärzte haben Sorgen, dass ihre ambulanten Versorgungsstrukturen zunehmend in die klinischen Strukturen eingebunden werden. Niedergelassene Kollegen, die mit ihrem privaten Vermögen für ihr Unternehmen auch haften, werden erst einmal abwarten, wie sich die ganze Sache weiterentwickelt. Auf der anderen Seite sieht es in den Kliniken auch nicht rosig aus, gerade hinsichtlich des SGB V. Wir sehen uns immer mehr bedroht durch ein um sich greifendes Honorararztwesen. Wir haben in den Kliniken einen Fachärztemangel und einen enormen ökonomischen Druck. In ganz besonderer Weise ist die Maximalversorgung mit ihren hohen Vorhaltekosten unterfinanziert.
Ob das neue Gesetz, das jetzt in der Diskussion ist, das sog. Versorgungsstärkungsgesetz, Löser dieser Probleme sein wird, sei dahingestellt. Im Referentenentwurf dieses Gesetzes mit 140 Seiten werden neue Konzepte formuliert, Themen wie Facharzttermine, ärztliche Zweitmeinung, Lösung der Unter- und Überversorgungskapazitäten, die Versorgung auf dem Land oder auch eine fraglich gerechtere und an Qualitätskriterien orientierte Vergütungsstruktur sollen dort adressiert werden. Wobei mir noch nicht ganz klar ist, ob die Versorgung nach Qualitätskriterien auch schon inhaltlich gefüllt ist. Auch ist noch gar nicht definiert, was Qualität eigentlich bedeutet und wie man Qualität misst. Es fehlt also noch an den Grundlagen, um eine Richtungsänderung herbeizuführen. Ob dadurch der Weg zu einem besseren und gerechteren Vergütungssystem nach SGB V geebnet wird, sei dahingestellt. Auf jeden Fall sind wir sehr gespannt.

Wie Herr Dr. Platz möchte ich noch auf zwei Termine dieser UMed hinweisen, nämlich auf die beiden Impulsreferate heute Nachmittag um 14 Uhr. Das erste Referat wird Herr Dr. Schüürmann halten. Er ist als niedergelassener Kollege in Hessen in einer Praxis tätig, sehr vernetzt als Vorsitzender des Hessischen Chirurgenverbandes und berufspolitisch sehr engagiert. Wir sind sehr gespannt auf sein Referat. Im zweiten Teil wird Herr Professor Winker, unser wissenschaftlicher Koleiter, ein Referat halten mit dem Titel „30 Jahre D-Arzt - ein Rückblick in die Zukunft“. Herr Professor Winker ist ein Thüringer mit schwäbischem Akzent. Nach langjähriger Tätigkeit in der BG-Klinik in Tübingen unter Professor Weller, Kooberarzt meines klinischen Lehrers Professor Wentzensen, ist er relativ kurz nach der Wende nach Erfurt gegangen und hat die Klinik dort über 20 Jahre lang geleitet. Er hat unzählige dankbare Patienten und ei- 
ne Vielzahl von Ärzten, die unter seiner Führung ausgebildet wurden, sozusagen durch seine Schule gegangen sind. Neben einer exzellenten medizinischen Ausbildung haben sie von ihm Menschlichkeit gegenüber Patienten und Abwägung zwischen Notwendigkeiten in der Medizin gegenüber den ökonomischen Zwängen in lebenslang prägender Weise gelernt. Er ist immer noch sehr aktiv in der Fort- und Weiterbildung von Ärzten international. Er ist ein sehr aktives Mitglied der AO international und er ist natürlich fachärztlicher Berater der DGUV und da dürfen wir durchaus stolz sein. Ich ziehe den Hut vor seiner Lebensleistung. Wir sind dankbar, dass er heute hier ist.

Ich möchte an dieser Stelle auch allen Referenten und Vorsitzenden für die Übernahme der Aufgaben bei der UMed danken, ganz besonders auch, dass Sie Ihre Beiträge dann verschriftlichen und uns dann in einigen Monaten auch wieder das schöne Heft zur Verfügung stehen wird, in dem Inhalte der UMed in Trauma und Berufskrankheit publiziert werden.

Ich möchte mich aber an dieser Stelle auch ganz explizit bei den Mitarbeitern des Landesverbandes Mitte der DGUV bedanken, die den wissenschaftlichen Leitern immer zur Seite standen, uns in jeder Hinsicht bei der Erstellung des Programms unterstützt haben. Ich denke, das ist eine große Anerkennung wert, dass mit einem relativ kleinen Team in einem Landesverband so eine Veranstaltung zustande kommt. Also vielen Dank dafür.

Ich wünsche uns jetzt abschließend eine spannende, interessante, erkenntnisreiche 32. Unfallmedizinische Tagung des Landesverbandes Mitte und ich danke Ihnen herzlich, dass Sie mir zugehört haben, und würde jetzt gleich die ersten Vorsitzenden bitten, die Sitzungsleitung zu übernehmen. Das wären Herr Professor Marzi und Herr Professor Ruchholtz, die direkt ins kindertraumatologische Thema einsteigen. Ich wünsche Ihnen eine schöne Tagung.

Prof. Dr. P.A. Grützner

\section{Korrespondenzadresse}

\section{Prof. Dr. P.A. Grützner}

Klinik für Unfallchirurgie und Orthopädie BG-Unfallklinik Ludwigshafen Ludwig-Guttmann-Str. 13, 67071 Ludwigshafen Paul.Gruetzner@bgu-ludwigshafen.de 